\title{
High-Cadence Transit Timing Variation Monitoring of Extrasolar Planets
}

\author{
V. D. Ivanov ${ }^{1}$, C. Cáceres ${ }^{2}$, D. Minniti², F. Selman ${ }^{1}$, C. Melo ${ }^{1}$, D. Naef ${ }^{3}$, E. \\ Mason $^{1}$, G. Pietrzynski ${ }^{4}$ \\ ${ }^{1}$ European Southern Observatory, Ave. Alonso de Córdova 3107, Casilla 19, \\ Santiago 19001, Chile [vivanov@eso.org]
}

${ }^{2}$ Department of Astronomy and Astrophysics, P. Universidad Catolica de Chile. Ave. Vicuña Mackenna 4860, 7820436 Macul, Santiago, Chile

${ }^{3}$ Observatoire de Genève, Université de Genève, 51 Ch. des Maillettes, 1290 Sauverny, Switzerland

${ }^{4}$ Departmento de Astronomía, Universidad de Concepción, Casilla 160-C, Concepcion, Chile

\begin{abstract}
We report ground-based high-cadence transit timing observations of the extrasolar planet WASP-2b. We achieve a typical timing error of 15-30 sec. The data show no significant deviations from the predicted ephemeris.
\end{abstract}

\section{Introduction}

The Transit Timing Variation (TTV; Agol et al. 2005) method uses the variation of the intervals between transits in the presence of a perturbing body to search for additional planets in systems with known transiting exoplanets. A finite number of photons is received during a transit, so high-cadence observations minimizing the loss to readout are needed. This is achieved either by defocusing the telescope (adding contamination from nearby stars and increasing the background) or by using detectors with low readout noise and minimal readout time - such as the ones in near-infrared instruments. We still had to hardware-window the detectors, reducing the minimum detector integration time (DIT) because short DITs are necessary to improve the time resolution, and to avoid saturating the bright sources. Our measurements are relative - we keep one or more reference source on the detector during the observations.

\section{Discussion and Results}

ISAAC@VLT and SOFI@NTT (Moorwood et al. 1998a, 1998b) were used in FASTPHOT mode. Windowing their detectors down to $512 \times 512$ pixels lowers the minimum DITs to $\sim 0.1$ and $\sim 0.6 \mathrm{sec}$, respectively.

The reference source for WASP-2 was at 2.58 arcmin away. The data reduction steps were: dark/bias subtraction with day-time darks, flat fielding with twilight flats, sky subtraction in circular annuli around the stars (observations were performed in stare mode to reduce the overheads), and aperture photometry within 5-10 arcsec diameter 

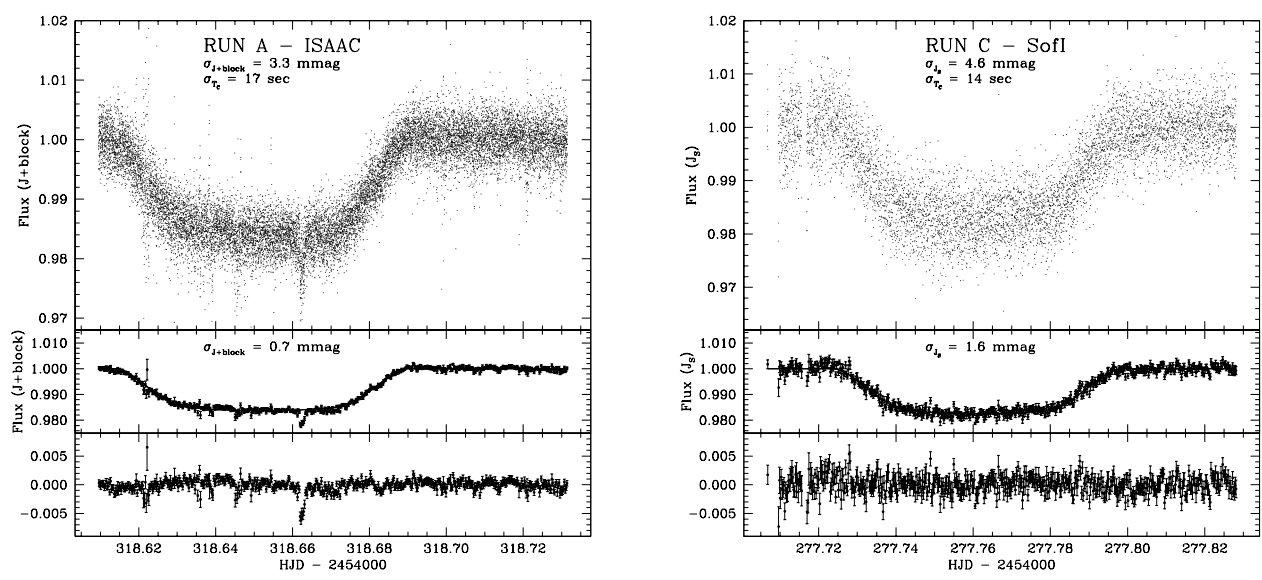

Figure 1.: Light curves (LC) of two WASP-2b transits from ISAAC (left) and SofI (right), with $86 \times 0.6 \mathrm{sec}$ and $800 \times 1.5 \mathrm{sec}$. The unbinned LCs (top) have RMS of 3.3 and $4.6 \mathrm{mmag}$, respectively. These are reduced to 0.7 and $1.6 \mathrm{mmag}$ for binning of $20 \mathrm{sec}$ (middle). The residuals for the binned LCs are shown at the bottom.

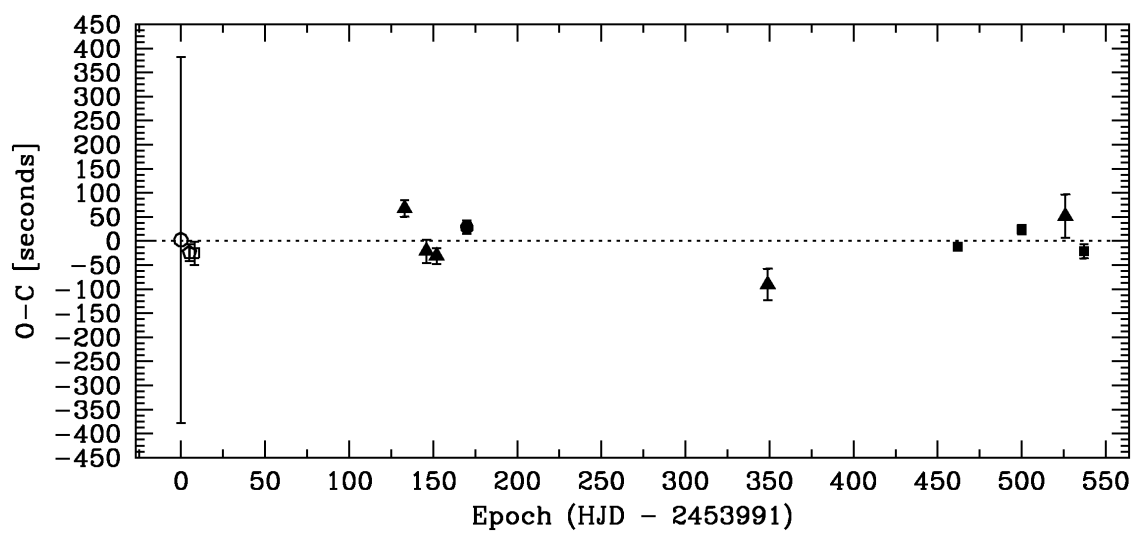

Figure 2.: Timing measurements for WASP-2b: solid triangles - this work, open circles - Collier-Cameron et al. (2007), stars - Wheatley et al. (2010), squares - Charbonneau et al. (2007), solid circles - Hrudkova et al. (2009), solid squares - Southworth et al. (2010). The ephemeris are from Southworth et al. (2010).

apertures. WASP-2b light curves (LC) are shown in Fig. 1. A contribution of $\sim 2 \%$ from a faint nearby companion 0.7 arcsec away from WASP-2b has not been removed. After a polynomial normalization to remove the largest systematics (i.e. airmass), the LC was fitted with analytical models from Mandel \& Agol (2002) via $\chi^{2}$ minimization. The timing error was determined with bootstrapping simulation, creating multiple artificial 
LCs combining the model fits and the real deviations, shifted in a circular fashion by a random number of steps (see Caceres et al. 2009).

Our five timing measurements (Fig. 2), with 90-95\% cadence, have timing accuracy of $\sim 15$-30 sec for an individual transit, and they show no indication for an additional body in this system.

\section{References}

Agol, E., Steffen, J., Sari, R., \& Clarkson, W. 2005, MNRAS, 359, 567

Cáceres, C., Ivanov, V.D., Minniti, D., Naef, D., Melo, C., Mason, E., Selman, F., \& Pietrzynski, G. 2009, A\&A, 507, 481

Charbonneau, D., Winn, J.N., Everett, M.E., Latham, D.W., Holman, M.J., Esquerdo, G.A., \& O’Donovan, F. T. 2007, ApJ, 658, 1322

Collier-Cameron, A., Wilson, D.M., West, R.G., et al. 2007, MNRAS, 380, 1230

Hrudkova, M., Skillen, I., Benn, C., Pollacco, D., Gibson, N., Joshi, Y., Harmanec, P., \& Tulloch, S. 2009, IAUS, 253, 477

Mandel, K., \& Agol, E. 2002, ApJ, 580, L171

Moorwood, A., Cuby, J.-G., Biereichel, P., et al. 1998a, The Messenger, 94, 7

Moorwood, A., Cuby, J.-G., \& Lidman, C. 1998b, The Messenger, 91, 9

Southworth, J., Mancini, L., Novati, S.C., et al. 2010, MNRAS, in press

Wheatley, P.J., Collier Cameron, A., Harrington, J., et al. 2010, ApJ, in press 